\title{
Postoperative Blood Pressure Could Be a Modifiable Risk Factor for Acute Kidney Injury After Coronary Bypass Surgery
}

\author{
Postoperatif Kan Basıncı Koroner Baypas Cerrahisi Sonrası Gelişen Akut Böbrek \\ Hasarının Düzenlenebilir Bir Risk Faktörü Olabilir
}

\author{
- Doğan Kahraman, ๑ Ozan Emiroğlu*
}

Gaziantep University Faculty of Medicine, Department of Cardiovascular Surgery, Gaziantep, Turkey

*Doktor Burhan Nalbantoğlu Hospital, Clinic of Cardiovascular Surgery, Lefkoşe, Turkish Republic of Northern Cyprus

\section{Abstract}

\begin{abstract}
Aim: Acute kidney injury (AKI) is a common complication associated with coronary surgery. This study aims to define perioperative modifiable risk factors for AKI development.

Methods: We prospectively observed perioperative parameters, laboratory tests, and outcome variables of 319 consecutive patients. The patients were divided into groups according to onpump or off-pump coronary bypass.

Results: The off-pump patients were older $(65.15 \pm 9.247$ years vs $60.81 \pm 9.659 ; p=0.001$ ) but he frequency of $A K I$ development was similar between the groups (22\% vs $19.9 \%, p=0.659)$. Preoperative hypertension, advanced age, high body weight, and prolonged cross-clamp time were associated with postoperative $\mathrm{AKI}$, but postoperative high blood pressure and higher ejection fraction were likely to be protective against AKI development. Expected in-hospital survival in a patient developing AKI after off-pump surgery was better than those with on-pump surgery ( $88.9 \%$ and $84.6 \%$, respectively; $p<0.001$ )
\end{abstract}

Conclusion: Managing postoperative blood pressure according to preoperative level could have the potential to reduce AKI incidence.

Keywords: Off-pump coronary artery bypass, acute kidney injury, blood pressure
Öz

Amaç: Akut böbrek hasarı $(A B H)$ koroner cerrahi sonrası sık görülebilen bir komplikasyondur. $\mathrm{Bu}$ çalışma $A B H$ gelişimi ile ilişkili perioperatif değiştirilebilir risk faktörlerini tanımlamayı amaçlamaktadır.

Yöntemler: Ardışık 319 hastanın perioperatif parametrelerini, laboratuvar testleri ve sonuç değişkenlerini prospektif olarak gözlemledik. Gruplar pompalı veya pompasız koroner bypass'a göre ayrıldı.

Bulgular: Pompasız koroner baypas hastaları daha yaşlıydı $(65,15 \pm 9,247$ ve $60,81 \pm 9,659 ; p=0,001)$, ancak $A B H$ gelişim sıklığı her iki grupta benzerdi (\%22 ve \%19,9; $p=0,659)$. Preoperatif hipertansiyon, yaş, yüksek vücut ağırlığı ve kros klemp süresi, postoperatif $A B H$ ile ilişkiliydi, ancak postoperatif yüksek kan basıncı ve yüksek ejeksiyon fraksiyonunun $A B H$ gelişimine karşı koruyucu faktörler olarak belirlendi. Postoperatif dönemde $\mathrm{ABH}$ gelişen bir hasta grubunda, pompasız ameliyatı sonrası hastane-sağkalımı, pompa ile baypas ameliyatı yapılanlara göre anlamlı derecede yüksekti (sırasıyla $\% 88,9$ ve $\% 84,6$; $p<0,001$ ).

Sonuç: Postoperatif kan basıncının preoperatif seviyeye göre yönetilmesi $\mathrm{ABH}$ gelişimini azaltma potansiyeline sahiptir.

Anahtar Sözcükler: Pompasız koroner arter baypası, akut börek hasarı, kan basıncı

\section{Introduction}

Acute kidney injury (AKI) after cardiac surgery is highly prevalent and is responsible for serious complications (1). Its incidence changes from $5 \%$ to $40 \%$ depending on various definitions (1-3). Even small increases from baseline in the serum creatinine $(\mathrm{s} C r)$ after cardiac surgery have been proposed to be associated with increased postoperative mortality $(4,5)$. It is unlikely that a single etiologic factor would cause perioperative AKI but it is
Address for Correspondence/Yazışma Adresi: Doğan Kahraman, Gaziantep University Faculty of Medicine, Department of Cardiovascular Surgery, Gaziantep, Turkey Phone: +90 3423606060 E-mail: drdogankahraman@gmail.com ORCID: orcid.org/0000-0002-8864-7065 Received/Geliş Tarihi: 27 September 2019 Accepted/Kabul Tarihi: 08 March 2020
${ }^{6}$ Copyright 2020 by The Medical Bulletin of istanbul Haseki Training and Research Hospital The Medical Bulletin of Haseki published by Galenos Yayınevi. ${ }^{\circ}$ Telif Hakkı 2020 İstanbul Haseki Eğitim ve Araştırma Hastanesi Haseki Tip Bülteni, Galenos Yayınevi tarafından yayınlanmıştır. 
rather a consequence of multiple and interactive pathways. The proposed risk factors, (ie. diabetes, age, chronic renal failure) are mostly constitutional and non-modifiable, therefore, have a limited significance in clinical practice (6). The favorable effect of off-pump coronary bypass (OPCAB) on renal injury is still controversial and various studies have compared outcomes between off-pump and on-pump coronary surgery (7). Though mostly retrospective, some reports indicated that AKI might be seen more frequently with on-pump coronary bypass (ONCAB), while others suggested that the difference between the procedures was not significant (6-8). Additionally, the preference of the surgeon between off-pump and on-pump surgery may change with preoperative risk factors such as comfort for distal anastomosis, ejection fraction, adverse outcomes related to cardiopulmonary bypass (CPB), etc.

Perioperative modifiable risk factors were scantily studied prospectively. This study aims to define perioperative hemodynamic parameters associated with AKI and to test the cumulative effect of CPB on mortality outcome related to kidney injury.

\section{Methods}

This observational study was approved by the institutional ethics committee of our hospital (Kavaklıdere Umut Hospital) at May 3rd 2012 (no: 2012/5-2). We prospectively analyzed the perioperative data of consecutive patients who underwent isolated coronary bypass grafting (CABG) between April 2012 and February 2013. Patients with end-stage renal disease with or without dialysis, $\mathrm{sCr}$ over $2.5 \mathrm{mg} / \mathrm{dL}$, concomitant aorta or valve surgery, and history of previous cardiac surgery were excluded. Informed consent was obtained from each patient and data analysis was conducted according to the declaration of Helsinki. The decision of on-pump or off-pump surgery was based on the preference of the surgeon, and a total of 319 patients were included (123 OPCAB patients and, 196 ONCAB patients). Preoperative demographic variables, laboratory test, hemodynamic parameters, arterial blood gas analysis, volume supplementation during fasting period, blood product use, number of bypass grafts, least urine yielded for 6-, 12- and 24-hr periods, and the results of surgery were recorded in a distinct follow-up chart for gathering data. Intraoperative hemodynamics with postoperative variables that can change the primary outcome of AKI was also followed for analysis.

\section{Assessment of Acute Kidney Injury}

AKI diagnosis was assessed according to the Acute Kidney Injury Network (AKIN) criteria (9). The last sCr value measured before surgery was defined as the baseline level. Presence and stages of new-onset AKI were calculated for each patient using baseline and the highest postoperative $\mathrm{s} C r$ level during the hospital stay. Urine volume $/ \mathrm{kg} / \mathrm{hr}$ was also calculated for the AKIN criterion by considering least urine harvest for 6 and 12 consequtive hours within the first 48 hours postoperatively. AKI is defined as an increase in $\mathrm{s} C r$ by $\geq 0.3 \mathrm{mg} / \mathrm{dL}$ within 48 hours; or an increase in $\mathrm{sCr}$ to $\geq 1.5$ times baseline, which is known or presumed to have occurred within the preceding seven days; or a urine volume $<0.5 \mathrm{~mL} / \mathrm{kg} / \mathrm{h}$ for 6 hours.

\section{Surgical Techniques}

All patients were operated through a median sternotomy. Graft preparation was followed by heparin bolus of 100-150 U/kg for OPCAB and $300 \mathrm{U} / \mathrm{kg}$ for ONCAB patients. Aortacaval cannulation, CPB induction and crossclamping were performed sequentially. The left internal thoracic artery was anastomosed only to the left anterior descending artery (LAD). The order of the anastomosis was the right coronary artery, the left circumflex, and the left anterior descending branch. The rectal temperature was kept between $30{ }^{\circ} \mathrm{C}$ and $33{ }^{\circ} \mathrm{C}$. The cross clamp was removed early after finishing distal bypasses and a side clamp was used for proximal anastomoses. CPB was terminated after checking distal anastomoses. Thoracic drains were inserted and the sternum was closed after ensuring bloodless surgical field.

In OPCAB surgery, exposure of target coronary artery and motionless surgical field required deep pericardial stay sutures and/or commercially available stabilizers. LIMA-to-LAD anastomosis was achieved first and this was subsequently followed by revascularization of the right coronary artery and left circumflex artery. Silicone rubber loops were routinely used before performing the relevant coronary anastomosis. Heparin bolus was not reversed, and sternum was closed after checking for bleeding.

\section{Statistical Analysis}

Continuous variables with normal distribution were expressed as mean \pm standard deviation and those without normal distribution as median (minimum-maximum). Categorical variables were expressed as number and percentage. To define significance level of differences between the groups, data were examined using the chisquare test or Fisher's exact test for dichotomous data, or Student's t-test and the Mann-Whitney $u$ test for perioperative continuous data whenever appropriate. The repeated measures ANOVA test was used to evaluate the changes in systolic blood pressure (SBP) in 3 different times after stratifying patients according to AKI development. We used Spearman's correlation coefficient to identify which variables were associated with AKI and among these variables with a $p$ value of $<0.20$, we selected covariates in binary logistic regression analysis considering known risk factors or relationships to hemodynamics perioperatively 
to determine independent risk factors for AKI. To avoid over-fitting of the model, we allowed a maximum of one variable per each 10 events. Model calibration was assessed using the Hosmer-Lemeshow goodness-of-fit test. A two-sided $p$ value of $<0.05$ was considered statistically significant. All statistical analyses were performed using the SPSS software, version 22.0 (IBM, Armonk, NY, USA).

\section{Results}

Of 341 patients enrolled, 22 were excluded from the analysis, because the patients had end-stage kidney disease requiring dialysis $(n=3)$, or had a history of cardiac surgery $(n=6)$, or ONCAB with concomitant valve or aorta surgery $(n=13)$. Three hundred nineteen patients (253 male and 66 female) with a mean age of $66.249 \pm 9.71$ years remained in this study. Except for older age in the OPCAB group $(p<0.01)$, the baseline characteristics of both groups and the laboratory findings were similar as shown in Table 1.

Results of univariate analysis of intraoperative variables are presented in Table 2 in which operative time was longer and number of patients needing transfusion and

\begin{tabular}{|c|c|c|c|c|c|c|c|}
\hline \multirow[t]{2}{*}{ Preoperative parameters } & \multicolumn{3}{|c|}{ OPCAB group $(n=123)$} & \multicolumn{3}{|c|}{ ONCAB group $(n=196)$} & \multirow[t]{2}{*}{$p$} \\
\hline & n (\%) & Mean \pm SD & Mean (min-max) & n (\%) & Mean \pm SD & Mean (min-max) & \\
\hline Age (yr) & - & $65.15 \pm 9.25$ & - & - & $60.81 \pm 9.66$ & - & 0.001 \\
\hline Geriatric ( $\geq 65 \mathrm{yr}$ ) & $54(43.9)$ & - & - & $59(30.1)$ & - & - & 0.012 \\
\hline Body weight (kg) & - & $80.76 \pm 8.93$ & - & - & $80.83 \pm 9.13$ & - & 0.948 \\
\hline \multicolumn{8}{|l|}{ Gender } \\
\hline Male & $94(76.4)$ & - & - & $159(81.8)$ & - & - & \multirow[t]{2}{*}{0.313} \\
\hline Female & $29(23.6)$ & - & - & 37 (18.9) & - & - & \\
\hline Hypertension & $67(54.5)$ & - & - & $112(57.1)$ & - & - & 0.640 \\
\hline ARB/ACEI use & $24(19.5)$ & - & - & $43(21.9)$ & - & - & 0.605 \\
\hline Diabetes Mellitus & $65(52.8)$ & - & - & $98(50)$ & - & - & 0.621 \\
\hline Metformin use & $29(23.6)$ & - & - & $50(25.5)$ & - & - & 0.697 \\
\hline Cerebrovascular event & $3(2.4)$ & - & - & 0 & - & - & 0.056 \\
\hline Ejection fraction (\%) & - & $54.27 \pm 8.53$ & - & - & $52.86 \pm 9.80$ & - & 0.192 \\
\hline CHF & $10(8.1)$ & - & - & $17(8.7)$ & - & - & 0.865 \\
\hline Atrial arrhythmia & $6(4.9)$ & - & - & $12(6.1)$ & - & - & 0.639 \\
\hline Ventriculerler arrhythmia & $4(3.3)$ & - & - & $3(1.5)$ & - & - & 0.436 \\
\hline Emergent surgery & $7(5.7)$ & & - & $6(3.1)$ & - & - & 0.248 \\
\hline BUN (mg/dL) & - & $17.54 \pm 4.80$ & - & - & $18.66 \pm 8.04$ & - & 0.163 \\
\hline $\mathrm{sCr}(\mathrm{mg} / \mathrm{dL})$ & - & $0.96 \pm 0.29$ & - & - & $0.99 \pm 0.25$ & - & 0.359 \\
\hline EGFR $\left(\mathrm{mL} / \mathrm{min} / 1.73 \mathrm{~m}^{2}\right)$ & - & $79.57 \pm 23.32$ & - & - & $78.45 \pm 20.41$ & - & 0.317 \\
\hline Urine Alb/Crea ratio & - & $24.76 \pm 17.34$ & - & - & $27.58 \pm 19.65$ & - & 0.188 \\
\hline Spot Urine protein $(\mathrm{mg} / \mathrm{dL})$ & - & - & $0.14(0.01-1.17)$ & - & - & $0.12(0.01-1.53)$ & 0.550 \\
\hline $\mathrm{pH}$ & - & $7.46 \pm 0.58$ & - & - & $7.45 \pm 0.45$ & - & 0.496 \\
\hline $\mathrm{pO}_{2}(\mathrm{mmHg})$ & - & $74.2 \pm 23.6$ & - & - & $71.7 \pm 20.3$ & - & 0.310 \\
\hline $\mathrm{pCO}_{2}(\mathrm{mmHg})$ & - & $33.7 \pm 6.4$ & - & - & $34.7 \pm 4.8$ & - & 0.097 \\
\hline $\mathrm{HCO}_{3^{-}}(\mathrm{mmol} / \mathrm{L})$ & - & $23.64 \pm 4.23$ & - & - & $24.70 \pm 3.55$ & - & 0.160 \\
\hline Sodium (mEq/L) & - & $141.2 \pm 3.8$ & - & - & $141.3 \pm 3.7$ & - & 0.858 \\
\hline Potassium (mEq/L) & - & $4.212 \pm 0.48$ & - & - & $4.217 \pm 0.50$ & - & 0.391 \\
\hline Hematocrit (\%) & - & $40.08 \pm 5.19$ & - & - & $40.27 \pm 4.46$ & - & 0.733 \\
\hline Total protein $(\mathrm{mg} / \mathrm{dL})$ & - & $6.93 \pm 0.66$ & - & - & $6.99 \pm 0.66$ & - & 0.362 \\
\hline Albumin (mg/dL) & - & $3.74 \pm 0.44$ & - & - & $3.81 \pm 0.40$ & - & 0.105 \\
\hline CRP (mg/L) & - & $13.12 \pm 8.93$ & - & - & $12.16 \pm 6.88$ & - & 0.285 \\
\hline Highest systolic BP (mmHg) & - & $125.66 \pm 19.98$ & - & - & $126.63 \pm 18.32$ & - & 0.659 \\
\hline Lowest Systolic BP (mmHg) & - & $101.8 \pm 14.0$ & - & - & $103.1 \pm 14.6$ & - & 0.410 \\
\hline Volume supplement $(\mathrm{mL})$ & - & $1124 \pm 458$ & - & - & $1088 \pm 417$ & - & 0.468 \\
\hline
\end{tabular}


the number of grafts used were significantly higher in ONCAB patients $(p<0.05)$. Approximately $20 \%$ of patients developed AKI within 2 days, and $40 \%$ of them kept remaining in stage 1 according to the AKIN criteria until discharge from hospital (Table 3). ONCAB group had more revision, more wound infection, more transfusion, and longer hospital stay.

The evaluation of the perioperative parameters of patients with AKI is presented in Table 4. Advanced age made a significant difference as an important risk factor in the OPCAB group $(70.93 \pm 7.36$ vs $63.36 \pm 9.03$, $p=0.001)$. Operative time was also longer in the ONCAB group as expected. In the repeated measures ANOVA test performed by stratifying the patients according to the development of AKI; SBP significantly decreased in all patients during the postoperative period. However, in the group developing AKI, the SBP decreased significantly more than in the group without AKI ( $p=0.001$, Figure 1).

The following variables were significantly associated with AKI in Spearman correlation analysis: age, body weight, ejection fraction, preoperative and postoperative highest SBP, Diabetes Mellitus, preoperative blood-ureanitrogen, preoperative and postoperative creatinine, intraoperative $\mathrm{O}_{2}$ saturation, postoperative C-reactive protein (CRP), postoperative albumin level, and urinary protein level. Besides these variables, operative time, cross-clamp time, and the number of packed blood transfusions were likely to increase the incidence of AKI (7). However, since urine output and creatinine levels were involved in the diagnosis of AKI, we thought creatinine and urine output to be clearly inappropriate for a risk factor to be analyzed by multivariate analysis considering model over-fitting. We added the 17 variables step by step into binary logistic regression analysis. Binary logistic regression analysis identified older age, higher body weight, preoperative high blood pressure, postoperative high CRP, operative time and cross-clamp time as independent risk factors for AKI (Table 5). However, high ejection fraction and high postoperative systolic BP were likely to be protective against AKI

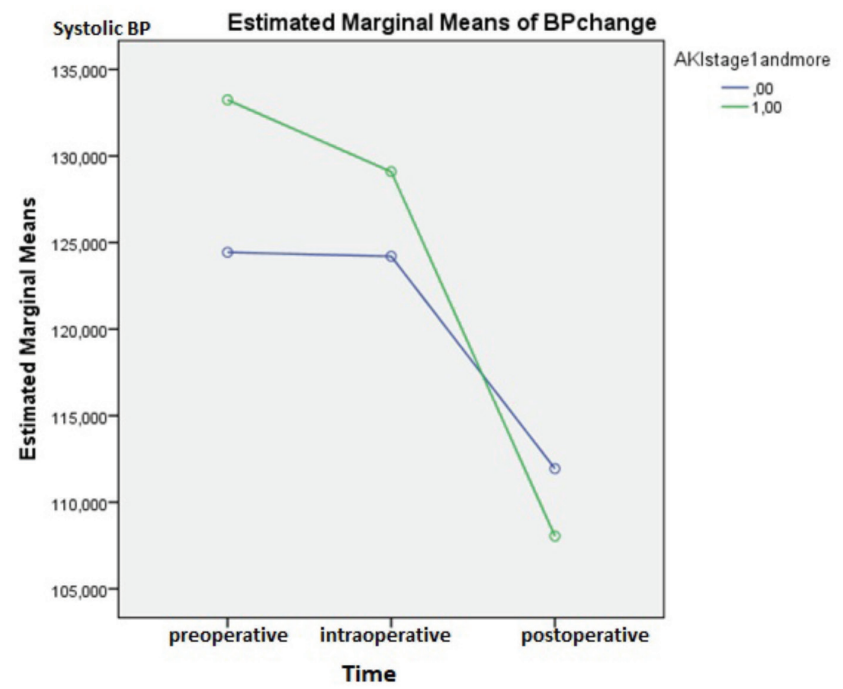

Figure 1. The schematic presentation of perioperative changes in systolic blood pressure after stratifying the patients according to AKI development. The repeated measures ANOVA test shows that both groups show significant decrease postoperatively $(p<0.01)$, and also AKI patients have more prominent decrease $(p<0.01)$. [Repeated measures ANOVA: Mapuchly's sphericity test; $p=0.001$, Greenhouse-Giesser $p=0.020$ Partial eta squared $=0.013, p$ value between the groups $<0.001$, $p$ value within the groups $<0.001$ ]

AKI: Acute kidney injury, BP: Blood pressure

\begin{tabular}{|c|c|c|c|c|c|c|c|}
\hline \multirow[t]{2}{*}{ Intraoperative parameters } & \multicolumn{3}{|c|}{ OPCAB group $(n=123)$} & \multicolumn{3}{|c|}{ ONCAB group $(n=196)$} & \multirow[t]{2}{*}{$p$} \\
\hline & n (\%) & Mean \pm SD & $\begin{array}{l}\text { Mean } \\
(\min -\max )\end{array}$ & n (\%) & Mean \pm SD & $\begin{array}{l}\text { Mean } \\
(\min -\max )\end{array}$ & \\
\hline $\begin{array}{l}\text { Systolic BP at induction of anesthesia } \\
(\mathrm{mmHg})\end{array}$ & - & $109.4 \pm 23.7$ & - & - & $108.35 \pm 24.9$ & - & 0.714 \\
\hline $\begin{array}{l}\text { Diastolic BP at induction of anesthesia } \\
(\mathrm{mmHg})\end{array}$ & - & $61.7 \pm 14.1$ & - & - & $63.9 \pm 14.7$ & - & 0.190 \\
\hline Heart rate at induction of anesthesia (bpm) & - & $72.7 \pm 14.5$ & - & - & $74.3 \pm 15.2$ & - & 0.352 \\
\hline Operation time ( $\mathrm{min})$ & - & $157.25 \pm 38.9$ & - & - & $204.57 \pm 56.56$ & - & 0.001 \\
\hline Cross-clamp time (min) & - & 0 & - & - & $51.19 \pm 17.67$ & - & - \\
\hline Patient needed transfusion & $53(43.1)$ & - & - & $146(74.5)$ & - & - & 0.001 \\
\hline New arrhythmia & $38(30.9)$ & - & - & $50(25.5)$ & - & - & 0.295 \\
\hline Lowest systolic BP (mmHg) & - & $62.93 \pm 17.95$ & - & - & $58.13 \pm 17.46$ & - & 0.019 \\
\hline Lowest diastolic BP $(\mathrm{mmHg})$ & - & $37.88 \pm 11.98$ & - & - & $39.86 \pm 12.52$ & - & 0.162 \\
\hline Lowest $\mathrm{O}_{2}$ saturation (\%) & - & - & $96(86-100)$ & - & - & $96(84-100)$ & 0.116 \\
\hline Number of graft & - & - & $2(1-3)$ & - & - & $4(2-5)$ & 0.001 \\
\hline
\end{tabular}


development. The Hosmer-Lemeshow goodness-of-fit test showed an adequate performance of the predictive model $\left(X^{2}=21,155, p=0.007\right)$.

We further performed Kaplan-Meier survival analysis by putting a stratum of type of surgery. Overall estimated survival during hospital stay was 97.2\%. However, estimated survival for OPCAB patients who developed AKI was significantly higher than for ONCAB patients with $\operatorname{AKI}\left(88.9 \%\right.$ and $84.6 \%$ respectively, $\left.X^{2}=36.412 ; p<0.001\right)$ (Figure 2).

\begin{tabular}{|c|c|c|c|c|c|c|c|}
\hline \multirow[t]{2}{*}{ Postoperative variables } & \multicolumn{3}{|c|}{ OPCAB group $(n=123)$} & \multicolumn{3}{|c|}{ ONCAB group $(n=196)$} & \multirow[t]{2}{*}{$\mathbf{p}$} \\
\hline & n (\%) & Mean \pm SD & $\begin{array}{l}\text { Mean } \\
(\text { min-max })\end{array}$ & n (\%) & Mean \pm SD & $\begin{array}{l}\text { Mean } \\
\text { (min-max) }\end{array}$ & \\
\hline$\geq$ Stage $1 \mathrm{AKI}$ at first $48 \mathrm{hr}$ & $27(22)$ & - & - & 39 (19.9) & - & - & 0.659 \\
\hline AKI stages at discharge & - & - & - & & - & - & 0.044 \\
\hline Stage 1 & $12(9.8)$ & - & - & $12(6.1)$ & - & - & - \\
\hline Stage 2 & $2(1.6)$ & - & - & 0 & - & - & - \\
\hline Stage 3 & $2(1.6)$ & - & - & 0 & - & - & - \\
\hline Oliguria & $19(15.4)$ & - & - & $14(7.1)$ & - & - & 0.018 \\
\hline Anuria & $5(4.1)$ & - & - & $2(1)$ & - & - & 0.112 \\
\hline Volume suppl// $1^{\text {st }}$ day $(\mathrm{mL})$ & - & $3575 \pm 814$ & - & - & $3502 \pm 684$ & - & 0.393 \\
\hline Least urine for $6 \mathrm{hr}(\mathrm{mL})$ & - & - & $450(175-850)$ & - & - & $420(150-830)$ & 0.801 \\
\hline Least urine for $12 \mathrm{hr}(\mathrm{mL})$ & - & - & $1075(400-2000)$ & - & - & $1050(530-1900)$ & 0.997 \\
\hline Urine volume $/ 1^{\text {st }}$ day $(\mathrm{mL})$ & - & $2586 \pm 862$ & - & - & $2516 \pm 762$ & - & 0.450 \\
\hline Urine $\mathrm{mL} / \mathrm{kg} / \mathrm{hr}$ within $1^{\text {st }}$ day & - & $1.37 \pm 0.56$ & - & - & $1.34 \pm 0.58$ & - & 0.608 \\
\hline Inotrope infusion (n) & $43(35)$ & - & - & $70(35.7)$ & - & - & 0.891 \\
\hline Vasodilator infusion (n) & $70(56,9)$ & - & - & $92(46.9)$ & - & - & 0.083 \\
\hline Lowest systolic BP (mmHg) & - & $94.14 \pm 19.18$ & - & - & $93.70 \pm 21.23$ & - & 0.854 \\
\hline Highest systolic BP $(\mathrm{mmHg})$ & & $139,0 \pm 27,2$ & & & $139.0 \pm 23.3$ & - & 0.977 \\
\hline Hypotensive attack (n) & $11(9,1)$ & & & $30(15,3)$ & - & - & 0.109 \\
\hline BUN (mg/dL) & - & $20.98 \pm 6.94$ & - & - & $22.37 \pm 8.47$ & - & 0.145 \\
\hline $\mathrm{sCr}(\mathrm{mg} / \mathrm{dL})$ & - & $1.07 \pm 0.65$ & - & - & $1.11 \pm 0.48$ & - & 0.514 \\
\hline BUN at discarge $(\mathrm{mg} / \mathrm{dL})$ & - & $20.71 \pm 7.78$ & - & - & $22.58 \pm 10.34$ & - & 0.087 \\
\hline $\mathrm{sCr}$ at discharge $(\mathrm{mg} / \mathrm{dL})$ & - & $0.96 \pm 0.29$ & - & - & $0.99 \pm 0.25$ & - & 0.359 \\
\hline Total protein $(\mathrm{mg} / \mathrm{dL})$ & - & $5.26 \pm 0.66$ & - & - & $5.24 \pm 0.61$ & - & 0.789 \\
\hline Albumin (mg/dL) & - & $2.65 \pm 0.32$ & - & - & $2.66 \pm 0.37$ & - & 0.674 \\
\hline Hematocrit (\%) & - & $27.33 \pm 3.60$ & - & - & $27.26 \pm 5.31$ & - & 0.891 \\
\hline CRP (mg/L) & - & $15.97 \pm 7.47$ & - & - & $16.34 \pm 6.81$ & - & 0.631 \\
\hline pH & - & $7.44 \pm 0.58$ & - & - & $7.43 \pm 0.75$ & - & 0.141 \\
\hline $\mathrm{pO}_{2}(\mathrm{mmHg})$ & - & $100.3 \pm 43.0$ & - & - & $101.0 \pm 41.1$ & - & 0.874 \\
\hline $\mathrm{pCO}_{2}(\mathrm{mmHg})$ & - & $36.3 \pm 8.2$ & - & - & $37.9 \pm 6.1$ & - & 0.048 \\
\hline $\mathrm{HCO}_{3}-(\mathrm{mmol} / \mathrm{L})$ & - & $26.84 \pm 3.44$ & - & - & $27.19 \pm 3.67$ & - & 0.391 \\
\hline $\mathrm{Na}(\mathrm{mEq} / \mathrm{L})$ & - & $140.4 \pm 3.8$ & - & - & 139.9 & - & 0.328 \\
\hline$K(\mathrm{mEq} / \mathrm{L})$ & - & $4.04 \pm 0.48$ & - & - & $4.02 \pm 0.44$ & - & 0.685 \\
\hline Total transfusion (pac) & - & - & $1(0-5)$ & - & - & $1(0-7)$ & 0.001 \\
\hline Revision & $6(4.9)$ & - & - & $20(10.2)$ & - & - & 0.091 \\
\hline Wound infection & $4(3.3)$ & - & - & $26(13.3)$ & - & - & 0.030 \\
\hline Readmission & $6(4.9)$ & - & - & $30(15.3)$ & - & - & 0.004 \\
\hline Exitus & $3(2.4)$ & - & - & $6(3.1)$ & - & - & 1 \\
\hline Postoperative hospital-stay (day) & - & - & $5(4-9)$ & - & - & $8.5(4-24)$ & 0.001 \\
\hline
\end{tabular}

OPCAB: Off-pump coronary bypass, ONCAB: On-pump coronary bypass, AKI: Acute kidney injury, BUN: Blood-urea-nitrogen, sCr: Serum creatinine, CRP: C-reactive protein, $\mathrm{pO}_{2}$ : Partial oxygen pressure, $\mathrm{pCO}_{2}$ : Partial carbondioxide pressure, $\mathrm{HCO}_{3}$ : Bicarbonate, Na: Sodium, $\mathrm{K}$ : Potassium, SD: Standard deviation, n: Number, BP: blood pressure 


\section{Discussion}

AKI is an abrupt loss of kidney function characterized by an increase in $\mathrm{s} C \mathrm{r}$ and regulated by a hyper-inflammatory state (10). Postoperative AKI is associated with increased in-hospital mortality and with significant readmission rate (11-13). Its incidence in patients with isolated CABG varies between $10 \%$ and $48.8 \%$ and, dialysis in an early postoperative period increases the risk of mortality up to $80 \%$ (14-16). Recent studies have focused on the pathogenesis of $\mathrm{AKI}$, but these have provided only a little innovative contribution to our clinical treatment strategy (14-17). Male gender, Diabetes Mellitus, age, hypertension, previous kidney disease, and poor EF are common risk factors (7). These risk factors are substantial and non-modifiable, but their clinical significance is limited.
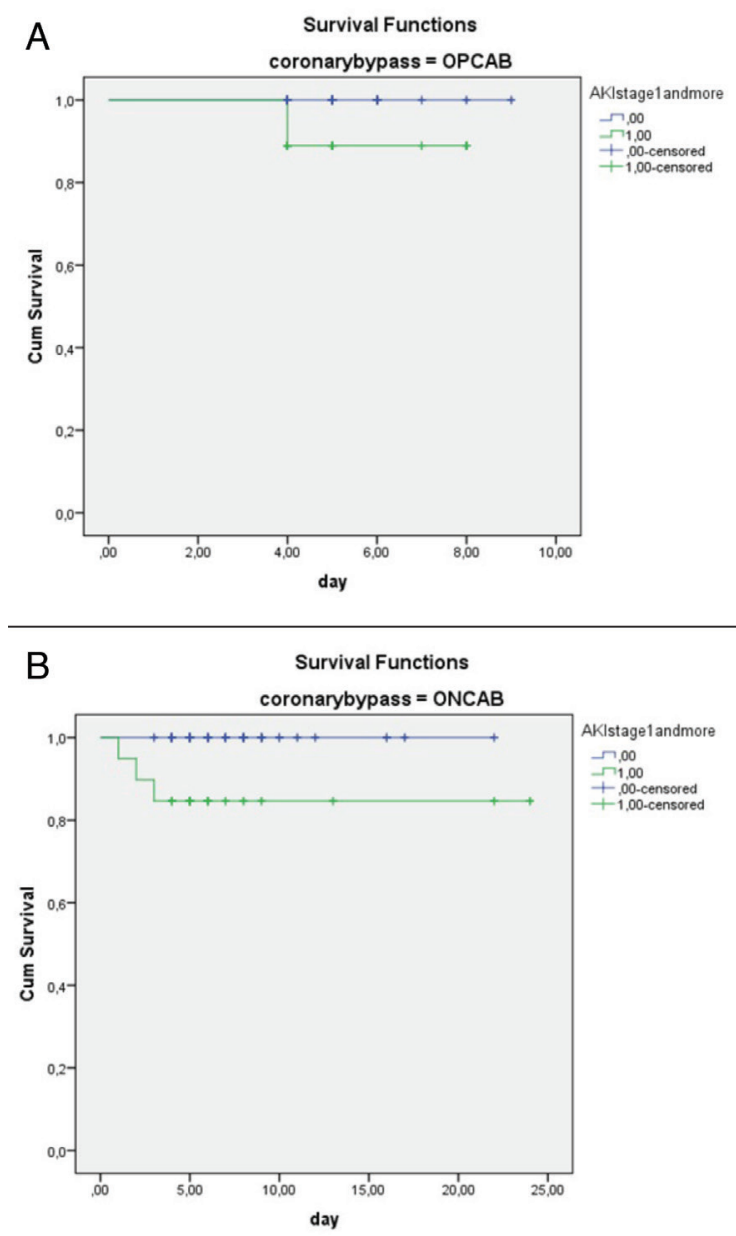

Figure 2. Demonstration of in-hospital survival after putting stratum of surgery types within the Kaplan-Meier analysis. The patients developing $\geq$ Stage $1 \mathrm{AKI}$ postoperatively had better survival rate after having OPCAB (A) than those undergoing ONCAB surgery (B)

OPCAB: Off-pump coronary bypass, ONCAB: On-pump coronary bypass, AKI: Acute kidney injury
Moreover, these non-modifiable risk factors might be curtaining the modifiable factors. There are accumulated reports in the literature to prove the association between demographic parameters and AKI development but patients' hemodynamics poses modifiable factors and may affect renal function acutely. However, hemodynamic factors have been rarely studied. We hypothesized some potentially modifiable predictors from previous studies and attenuated the influence of the confounding factors such as demographics and baseline clinical parameters by limiting patient selection criteria $(12,14,15,17,18)$. In clinical practice, the accurate risk prediction of AKI can help us identify high-risk patients for more effective prevention and timely treatment.

The present study revealed that the incidence of $A K I$ after $O P C A B$ and $O N C A B$ surgeries are $22 \%$ and $19.9 \%$, respectively. Amongst these patients, 3.5\% needed renal replacement therapy, and the mortality rate was $13.6 \%$. The results of this study demostrated that age, body weight, EF, preoperative hypertension, operative time, cross-clamp time, and postopreative systolic BP were associated with development of AKI. Amongst these, the cross-clamp time was the most powerfull independent risk factor ( $r r=5.3$ ), since it indirectly reflects the CPB that is a non-pyhsiologic condition set for ONCAB.

In the isolated CABG cohort, the preoperative highest systolic BP was evaluated to discriminate patients with uncontrolled hypertension and it was found to be a risk factor, whereas postoperative highest BP was protective against AKI. We think that patients with uncontrolled hypertension are more likely to have a decreased arterial elasticity and propensity for atherosclerosis, which would aggravate hypoperfusion during the postoperative period. Concordantly, several previous studies have shown that BP reduction was associated with an increased incidence of AKI in patients with acute decompensated heart failure (1922). Indeed, our patient cohort was not decompensated heart failure but cross-clamping in ONCAB and localized myocardial ischemia during OPCAB might potentially result in temporarily altered ventricular function (23-26). Frequent use of inotropes and antihypertensive infusion to stabilize BP within optimal levels reflects abrupt changes in hemodynamic parameters together with cardiac functions, though ideal levels of BP may change individually and among different clinics. Maintaining the optimum cardiac output is an important aspect of preventing $\mathrm{AKI}$, especially for vasopressor-dependent patients $(24,26)$. Although mean arterial pressure has played an important role in preventing AKI in previous trials, there is little evidence showing that instant systolic BP actually reflects renal perfusion. Postoperative BP change makes a significant difference in patients with AKI 
Kahraman and Emiroğlu. Renal Injury After On-pump is More Mortal

\begin{tabular}{|c|c|c|c|c|c|}
\hline \multirow[t]{2}{*}{ AKI developing patients } & \multicolumn{2}{|c|}{ OPCAB group $(n=27)$} & \multicolumn{2}{|c|}{ ONCAB group $(n=39)$} & \multirow[t]{2}{*}{$p$} \\
\hline & Mean \pm SD & Mean (min-max) & Mean \pm SD & Mean (min-max) & \\
\hline Age (yr) & $70.93 \pm 7.36$ & - & $63.36 \pm 9.03$ & - & 0.001 \\
\hline Body weight (kg) & $85.89 \pm 8.04$ & - & $85.05 \pm 8.70$ & - & 0.693 \\
\hline Preoperative sysytolic BP (mmHg) & $135.9 \pm 24.2$ & - & $131.4 \pm 20.1$ & - & 0.410 \\
\hline Preoperative BUN (mg/dL) & - & $18(13-27)$ & - & $19(12-64)$ & 0.036 \\
\hline Preoperative creatinin (mg/dL) & - & $0.9(0.5-1.5)$ & - & $1.1(0.7-2.1)$ & 0.139 \\
\hline Preoperative $\mathrm{pH}$ & $7.46 \pm 0.06$ & - & $7.44 \pm 0.04$ & - & 0.143 \\
\hline Preoperative $\mathrm{Na}(\mathrm{mEq} / \mathrm{L})$ & $141.7 \pm 4.69$ & - & $140.6 \pm 4.36$ & - & 0.354 \\
\hline Preoperative $\mathrm{K}$ (mEq/L) & $4.21 \pm 0.33$ & - & $4.32 \pm 0.51$ & - & 0.306 \\
\hline Preoperative albümin (mg/dL) & $3.8 \pm 0.30$ & - & $3.7 \pm 0.47$ & - & 0.592 \\
\hline Intraoperative systolic BP (mmHg) & $117.1 \pm 36.12$ & - & $113.9 \pm 27.5$ & - & 0.057 \\
\hline Operation time (min) & - & $152(50-215)$ & - & $208(92-370)$ & 0.001 \\
\hline Postoperative systolic BP (mmHg) & $111.9 \pm 15.1$ & - & $113.9 \pm 27.5$ & & - \\
\hline Postoperative BUN (mg/dL) & - & $25(19-35)$ & - & $28(16-57)$ & 0.364 \\
\hline Postoperative creatinin (mg/dL) & - & $1.4(0.9-3.6)$ & - & $1.5(1-3.8)$ & 0.651 \\
\hline Postoperative $\mathrm{pH}$ & $7.44 \pm 0.57$ & - & $7.47 \pm 0.74$ & - & 0.330 \\
\hline Postoperative $\mathrm{Na}(\mathrm{mEq} / \mathrm{L})$ & $140.1 \pm 2.89$ & - & $140.2 \pm 3.81$ & - & 0.961 \\
\hline Postoperative K (mEq/L) & $4.07 \pm 0.39$ & - & $4.08 \pm 0.45$ & - & 0.908 \\
\hline Postoperative albümin (mg/dL) & $2.53 \pm 0.26$ & - & $2.62 \pm 0.36$ & - & 0.274 \\
\hline
\end{tabular}

Table 5. The factors that had a significant correlation with AKI were analyzed for possible predictive effect on the development of postoperative AKI. Eight factors having significant predictive value with regression analysis are demonstrated

\begin{tabular}{|l|l|l|l|l|l|}
\hline & Spearman correlation & \multicolumn{3}{l|}{ Binary logistic regression } \\
\cline { 2 - 6 } & $\mathbf{C C}$ & $\mathbf{p}$ & $\mathbf{R R}$ & $\mathbf{9 5 \%}$ CI & $\mathbf{p}$ \\
\hline Age & $0.197^{* *}$ & 0.000 & 1.084 & $1.026-1.145$ & 0.004 \\
\hline EF & -0.099 & 0.077 & 0.934 & $0.883-0.987$ & 0.015 \\
\hline Body weight & $0.261^{* *}$ & 0.000 & 1.202 & $1.129-1.280$ & 0.000 \\
\hline Preoperative highest BP & $0.169^{* *}$ & 0.003 & 1.038 & $1.015-1.061$ & 0.001 \\
\hline Postoperative CRP & $0.181^{* *}$ & 0.001 & 1.064 & $1.009-1.123$ & 0.023 \\
\hline Postoperative highest BP & -0.043 & 0.440 & 0.974 & $0.949-1.000$ & 0.049 \\
\hline Operation time & $0.246^{* *}$ & 0.000 & 1.002 & $1.001-1.003$ & 0.000 \\
\hline X-clamp time & 0.080 & 0.156 & 5.347 & $1.245-22.727$ & 0.024 \\
\hline $\begin{array}{l}\text { AKI: Acute kidney injury, CC: Correlation coefficient, RR: Relative risk, Cl: Confidence interval, EF: Ejection fraction, BP: Blood pressure, CRP: C-reactive protein } \\
\text { Model summary: (-2 Log likelihood=178.724) (Cox\&Snell R2=0.357). Hosmer-Lemeshow:X2=21.155 and p=0.007 }\end{array}$ \\
\hline
\end{tabular}

(Figure 1). Considering the gap in the slope of decrease, there might be 2-way inference. First, as seen in Figure 1, the mean preoperative BP in patients who developed AKI was higher than in those who did not. It can be thought that perioperative normalization of $\mathrm{BP}$ in these patients poses a risk for the development of $\mathrm{AKI}$. Or, keeping the BP low in the postoperative period due to fear of excessive bleeding without considering the preoperative BP may be thought to increase the risk of developing AKI.

Some studies suggest that CPB time is an important contributor to postoperative renal dysfunction (27). However, we used the cross-clamp time to compare the purifying effect of elective on-pump and off-pump coronary surgeries in which cross-clamp time has a significant correlation with CPB time and operative time $(27,28)$. An increase in cross-clamp time prolongs hypoperfusion of renal medulla and may also show remote effects of ischemia-reperfusion injury. Besides, patients who undergo ONCAB often have altered left ventricular systolic functions, and they are more likely to receive potent drugs that may affect renal perfusion and the sCr level. Also, increased body weight (obesity) is associated with postoperative AKI, similar to previously published reports. We know that obesity 
increases oxidative stress, endothelial dysfunction, and inflammation (29). Though the mean operative time in both groups was within acceptable limits, it was also related to AKI development as reported in the literature $(12,13)$. The rate of development of AKI in both groups did not differ in the present study. The differences in age, in favor of the OPCAB group possibly covered all the negative effects of cross-clamp, amount of blood transfusion, operative time, and CPB on renal function.

Initially, we compared the in-hospital survey by dividing patients into with or without development of kidney injury. In fact, all mortal cases had an elevated $\mathrm{sCr}$ level from the baseline level. This was expected but useless to infer a conclusion. We put a stratum of type of surgery and the result showed that amongst patients developing $\geq$ stage $1 \mathrm{AKI}, \mathrm{OPCAB}$ group had significantly better survival than the ONCAB patients. The cumulative effect of cross-clamping overweighted the comfort for more delicate distal anastomosis, therefore, we infer that AKI development after ONCAB is more mortal than after OPCAB.

Most renal dysfunction after cardiac surgery is a temporary and reversible event. Patients with mild AKI are usually responsive to medical management and show spontaneous recovery. In our study, patients with stage 1 AKI showed an increased level of $\mathrm{s} C r$ in the first 2 days but frequently returned to the baseline level during hospital stay. A prompt intervention in postoperative management, especially avoiding additional renal insult, managing BP accordingly and optimizing volume status, can prevent further progression of perioperative AKI, and occurrence of worst outcomes, including hospital mortality. Thus, intensive renal preservation during perioperative period appears to provide sufficient renal protection.

Further studies are needed to determine how to optimize cardiac surgical procedures, intraoperative or early postoperative BP and renal blood flow to reduce the risk of AKI. No single pharmacologic agent has demonstrated clear efficacy in the prevention of AKI (30); however, postoperative BP managed near to preoperative baseline systolic BP may show promising results in renoprotection.

\section{Study Limitations}

Though preoperative demographics of the patient cohort were similar, age of the patients became an effective criterion on surgeons' preference while deciding off-pump surgery. Since it is also a risk factor for AKI, age should be a confounding factor for inferences from the study population (7). The incidence of AKI development insignificantly differs although the operative time indicating the period of hypoperfusion was longer in the OPCAB group. Therefore, randomization considering constitutional factors may result in more frequent $\mathrm{AKI}$ in the on-pump group. The length of hypotensive period is also an important factor to determine the depth of renal hypoperfusion perioperatively. Although we did not determine the length of hypotension, the time-averaged evaluation of BP would contribute to a more powerfull inferences about our proposal.

\section{Conclusion}

The rate of postoperative AKI development insignificantly differs between on-pump and off-pump coronary surgeries. Besides constitutional factors, which are usually non-modifiable, postoperative BP management considering preoperative measurements may be clinically useful in concern to preclude kidney injury. However, AKI after ONCAB may result in a significantly higher increase in the risk of mortality than those after OPCAB.

\section{Authorship Contributions}

Concept: D.K. Design: D.K. Data Collection or Processing: D.K., O.E. Analysis or Interpretation: D.K. Literature Search: O.E. Writing: D.K.

Conflict of Interest: No conflict of interest was declared by the authors.

Financial Disclosure: The authors declared that this study received no financial support.

\section{References}

1. Maitra G1, Ahmed A, Rudra A, Wankhede R, Sengupta S, Das T. Renal dysfunction after off-pump coronary artery bypass surgery- risk factors and preventive strategies. Indian J Anaesth 2009;53:401-7.

2. Vives $M$, Wijeysundera $D$, Marczin $N$, et al. Cardiac surgeryassociated acute kidney injury. Interact Cardiovasc Thorac Surg 2014;18:637-45.

3. Wang Y, Bellomo R. Cardiac surgery-associated acute kidney injury: Risk factors, pathophysiology and treatment. Nature Reviews Nephrology 2017;13:697-711.

4. Mangano CM, Diamondstone LS, Ramsay JG, et al. Renal dysfunction after myocardial revascularization: risk factors, adverse outcomes, and hospital resource utilization. The Multicenter Study of Perioperative Ischemia Research Group. Ann Intern Med 1998;128:194-203.

5. Brown JR, Kramer RS, Coca SG, et al. Duration of acute kidney injury impacts long-term survival after cardiac surgery. Ann Thorac Surg 2010;90:1142-8.

6. Chertow GM, Lazarus JM, Christiansen CL, et al. Preoperative renal risk stratification. Circulation 1997;95:878-84.

7. Rosner MH, Okusa MD. Acute kidney injury associated with cardiac surgery. Clin J Am Soc Nephrol 2006;1:19-32.

8. Thakar CV, Arrigain S, Worley S, Yared JP, Paganini EP. A clinical score to predict acute renal failure after cardiac surgery. J Am Soc Nephrol 2005;16:162-8. 
9. Koza Y. Acute kidney injury: current concepts and new insights. J Inj Violence Res 2016;8:58-62.

10. Wang Y, Feng F, Liu M, Xue J, Huang $H$. Resveratrol ameliorates sepsis-induced acute kidney injury in a pediatric rat model via Nrf2 signaling pathway. Exp Ther Med 2018;16:3233-40.

11. Brown JR, Parikh CR, Ross CS, et al. Impact of perioperative acute kidney injury as a severity index for thirty-day readmission after cardiac surgery. Ann Thorac Surg 2014;97:111-7.

12. Brown JR, Hisey WM, Marshall EJ, et al. Acute Kidney Injury Severity and Long-Term Readmission and Mortality After Cardiac Surgery. Ann Thorac Surg 2016;102:1482-9.

13. An R, Pang QY, Liu HL. Association of intra-operative hypotension with acute kidney injury, myocardial injury and mortality in non-cardiac surgery: A meta-analysis. Int J Clin Pract 2019;23:e13394.

14. Amini S, Najafi MN, Karrari SP, et al. Risk Factors and Outcome of Acute Kidney Injury after Isolated CABG Surgery: a Prospective Cohort Study. Braz J Cardiovasc Surg 2019;34:705.

15. Hertzberg D, Sartipy U, Lund LH, Rydén L, Pickering JW, Holzmann MJ. Heart failure and the risk of acute kidney injury in relation to ejection fraction in patients undergoing coronary artery bypass grafting. Int J Cardiol 2019;274:66-70.

16. Felicio ML, Andrade RR, Castiglia YM, Silva MA, Vianna PT, Martins AS. Cystatin C and glomerular filtration rate in the cardiac surgery with cardiopulmonary bypass. Rev Bras Cir Cardiovasc 2009;24:305-11.

17. Wu Q, Yang $H$, Bo $H$, et al. Predictive role of estimated glomerular filtration rate prior to surgery in postsurgical acute kidney injury among very elderly patients: a retrospective cohort study. Ren Fail 2019;41:866-74.

18. Xu Z, Luo Y, Adekkanattu P, et al. Stratified Mortality Prediction of Patients with Acute Kidney Injury in Critical Care. Stud Health Technol Inform 2019;264:462-6.

19. Bangalore S, Qin J, Sloan S, Murphy SA, et al. What is the optimal blood pressure in patients after acute coronary syndromes?: Relationship of blood pressure and cardiovascular events in the pravastatin or atorvastatin evaluation and infection therapy-thrombolysis In myocardial infarction (PROVE IT-TIMI) 22 trial. Circulation 2010;122:2142-51.
20. Wohlfahrt $P$, Krajcoviechova A, Jozifova $M$, et al. Low blood pressure during the acute period of ischemic stroke is associated with decreased survival. J Hypertens 2015; 33:339-45.

21. Badin J, Boulain T, Ehrmann S, et al. Relation between mean arterial pressure and renal function in the early phase of shock: a prospective, explorative cohort study. Crit Care 2011;15:R135.

22. Arao Y, Sawamura A, Nakatochi M, et al. Early Blood Pressure Reduction by Intravenous Vasodilators Is Associated with Acute Kidney Injury in Patients with Hypertensive Acute Decompensated Heart. Circ J 2019;83:1883-90.

23. Xu S, Zhang J, Xu YL, Wu HB, Xue XD, Wang HS. Relationship between Angiotensin Converting Enzyme, Apelin, and NewOnset Atrial Fibrillation after Off-Pump Coronary Artery Bypass Grafting. Biomed Res Int 2017;2017:7951793.

24. Rosner MH, Okusa MK. Acute kidney injury associated with cardiac surgery. Clin J Am Nephrol 2006;1:19-32.

25. Timurkaynak T. Renin inhibisyonu nedir? Etki mekanizması. Arch Turk Soc Cardiol 2009;37(Suppl 7):5-14.

26. Koene RJ, Kealhofer JV, Adabag S, Vakil K, Florea VG. Effect of coronary artery bypass graft surgery on left ventricular systolic function. J Thorac Dis 2017;9:262-70.

27. Karim HM, Yunus M, Saikia MK, Kalita JP, Mandal M. Incidence and progression of cardiac surgery-associated acute kidney injury and its relationship with bypass and cross clamp time. Ann Card Anaesth 2017;20:22-7.

28. Thongprayoon C, Cheungpasitporn W, Lin J, Mao MA, Qian Q. Acute kidney injury in octogenarians after heart valve replacement surgery: a study of two periods over the last decade. Clin Kidney J 2017;10:648-54.

29. Scicchitano P, Cortese F, Gesualdo M, De Palo M, Massari F, Giordano P, Ciccone MM. The role of endothelial dysfunction and oxidative stress in cerebrovascular diseases. Free Radic Res 2019;53:579-95.

30. Yuan SM. Acute Kidney Injury after Cardiac Surgery: Risk Factors and Novel Biomarkers. Braz J Cardiovasc Surg 2019;34:352-60. 